Mathematical Modelling and Analysis

Volume 21 Number 1, January 2016, 83-94

http://dx.doi.org/10.3846/13926292.2016.1136901

(C) Vilnius Gediminas Technical University, 2016
Publisher: Taylor\&Francis and VGTU

http://www.tandfonline.com/TMMA

ISSN: $1392-6292$

eISSN: 1648-3510

\title{
Self-Similar Analytic Solution of the Two-Dimensional Navier-Stokes Equation with a Non-Newtonian Type of Viscosity
}

\author{
Imre Ferenc Barna ${ }^{a}$, Gabriella Bognár ${ }^{b}$ \\ and Krisztián Hriczó ${ }^{b}$ \\ ${ }^{a}$ Wigner Research Center of the Hungarian Academy of Sciences \\ Konkoly-Thege út 29-33, 1121 Budapest, Hungary \\ ${ }^{b}$ University of Miskolc \\ 3515 Miskolc-Egyetemváros, Hungary \\ E-mail(corresp.): mathk@uni-miskolc.hu \\ E-mail: barna.imre@wigner.mta.hu
}

Received May 15, 2015; revised December 22, 2015; published online January 15, 2016

\begin{abstract}
We investigate Navier-Stokes (NS) and the continuity equations in Cartesian coordinates and Eulerian description for the two dimensional incompressible nonNewtonian fluids. Due to the non-Newtonian viscosity we consider the Ladyzenskaya model with a non-linear velocity dependent stress tensor. The key idea is the multidimensional generalization of the well-known self-similar Ansatz, which has already been used for non-compressible and compressible viscous flow studies. Geometrical interpretations of the trial function are also discussed. Our recent results are compared to the former Newtonian ones.
\end{abstract}

Keywords: self-similar solution, Ladyzenskaya model, non-Newtonian fluid.

AMS Subject Classification: 34B09; 35Q30.

\section{Introduction}

Dynamical analysis of viscous fluids is a never-ending crucial problem. A large part of real fluids do not strictly follow Newton's law and are aptly called nonNewtonian fluids. In most cases, fluids can be described by more complicated governing rules, which means that the viscosity has some additional density, velocity or temperature dependence or even all of them. General introductions to the physics of non-Newtonian fluid can be found in $[2,12]$. In the following, we will examine the properties of a Ladyzenskaya type non-Newtonian fluid $[3,21]$. Additional temperature or density dependent viscosities will not be considered in the recent study. There are numerous analytical studies available for non-Newtonian flows, which shows some similarity to our recent problem [7, 
$8,15,16,17,20,23]$. The heat transfer in the boundary layer of a non-Newtonian Ostwald-de Waele power law fluid was investigated with self-similar Ansatz from one of us in details as well [11]. We use the two-dimensional generalization of the well-known self-similar Ansatz [4,22,25], which was already used to investigate the three dimensional non-compressible Newtonian NS [5] and the compressible Newtonian NS [6] equations. We compare our results to the former Newtonian cases. In the last part of our manuscript we present a series solution for the ordinary differential equation (ODE), which was obtained with the help of the self-similar Ansatz.

\section{Theoretical background}

The Ladyzenskaya [21] model of non-Newtonian fluid dynamics can be formulated in the most general vectorial form of

$$
\begin{aligned}
& \rho \frac{\partial \mathbf{u}_{i}}{\partial t}+\rho \mathbf{u}_{j} \frac{\partial \mathbf{u}_{i}}{\partial x_{j}}=-\frac{p}{\partial \mathbf{x}_{i}}+\frac{\partial \Gamma_{i j}}{\partial \mathbf{x}_{j}}+\rho \mathbf{a}_{i}, \\
& \frac{\partial \mathbf{u}_{j}}{\partial \mathbf{x}_{j}}=0 \\
& \Gamma_{i j} \stackrel{D e f}{=}\left(\mu_{0}+\mu_{1}|E(\nabla \mathbf{u})|^{r}\right) E_{i j}(\nabla \mathbf{u}), \\
& E_{i j}(\nabla \mathbf{u}) \stackrel{D e f}{=} \frac{1}{2}\left(\frac{\partial \mathbf{u}_{i}}{\partial \mathbf{x}_{j}}+\frac{\partial \mathbf{u}_{j}}{\partial \mathbf{x}_{i}}\right)
\end{aligned}
$$

where $\rho, \mathbf{u}_{i}, p, \mathbf{a}_{i}, \mu_{0}, \mu_{1}, r$ are the density, the two dimensional velocity field, the pressure, the external force, the dynamical viscosity, the consistency index and the flow behavior index. The last one is a dimensionless parameter of the flow. (To avoid further misunderstanding, we use a for external field instead of the letter $g$, which is reserved for a self-similar solution.) The $E_{i j}$ is the Newtonian linear stress tensor, where $\mathbf{x}(\mathrm{x}, \mathrm{y})$ are the Cartesian coordinates. The Einstein summation is used for the $\mathrm{j}$ subscript. In our next model, the exponent should be $r>-1$. This general description incorporates the following five different fluid models: Newtonian for $\mu_{0}>0, \mu_{1}=0$, Rabinowitsch for $\mu_{0}, \mu_{1}>0$, $r=2$, Ellis for $\mu_{0}, \mu_{1}>0, r>0$, Ostwald-de Waele for $\mu_{0}=0, \mu_{1}>0$, $r>-1$, Bingham for $\mu_{0}, \mu_{1}>0, r=-1$. For $\mu_{0}=0$ if $r<0$ then a fluid is called a pseudo-plastic fluid, if $r>0$ it is a dilatant fluid [12].

In pseudoplastic or shear thinning fluid, the apparent viscosity decreases with increased stress. Examples are: nail polish, whipped cream, ketchup, molasses, syrups, latex paint, ice, blood, some silicone oils, some silicone coatings and paper pulp in water. For the paper pulp, the numerical Ostwald-de Waele parameters are $\mu_{1}=0.418, r=-0.425$ [12]. Parameters of a film foam with carbon dioxides are $\mu_{1}=0.15, r=-0.52$ [14].

In dilatant or shear thickening fluid, the apparent viscosity increases with increased stress. Typical examples are suspensions of corn starch in water (sometimes called oobleck) or sand in water.

We set the external force to zero in our investigation $\mathbf{a}_{\mathbf{i}}=0$. In two dimensions, the absolute value of the stress tensor reads:

$$
|E|=\left[u_{x}^{2}+v_{y}^{2}+1 / 2\left(u_{y}+v_{x}\right)^{2}\right]^{1 / 2},
$$


where the $\mathbf{u}(u, v)$ coordinate notation is used from now on. For a better transparency - instead of the the usual partial derivation notation - we apply subscripts for partial derivations. (Note, that in three dimensions the absolute value of the stress tensor would be much complicated containing six terms instead of three.)

Introducing the following compact notation

$$
L=\mu_{0}+\mu_{1}|E|^{r}
$$

our complete two dimensional NS system for incompressible fluids can be formulated much clearer:

$$
\begin{aligned}
& u_{x}+v_{y}=0, \\
& u_{t}+u u_{x}+v u_{y}=-p_{x} / \rho+L_{x} u_{x}+L u_{x x}+\frac{L_{y}}{2}\left(u_{y}+v_{x}\right)+\frac{L}{2}\left(u_{y y}+v_{x y}\right), \\
& v_{t}+u v_{x}+v v_{y}=-p_{y} / \rho+L_{y} v_{y}+L v_{y y}+\frac{L_{x}}{2}\left(u_{y}+v_{x}\right)+\frac{L}{2}\left(v_{x x}+u_{x y}\right),
\end{aligned}
$$

which is our starting point for the next investigation.

We apply the generalized physically relevant self-similar Ansatz to system (2.2). The original form of the one-dimensional self-similar Ansatz is given in $[4,22,25]$

$$
T(x, t)=t^{-\alpha} f\left(\frac{x}{t^{\beta}}\right):=t^{-\alpha} f(\omega),
$$

where $T(x, t)$ can be an arbitrary variable of a partial differential equation (PDE) and $t$ means time and $x$ means spatial dependence. The similarity exponents $\alpha$ and $\beta$ are of primary physical importance since $\alpha$ represents the rate of decay of the magnitude $T(x, t)$, while $\beta$ is the rate of spread (or contraction if $\beta<0$ ) of the space distribution for $t>0$. The most powerful result of this Ansatz is the fundamental or Gaussian solution of the Fourier heat conduction equation (or for Fick's diffusion equation) with $\alpha=\beta=1 / 2$. This transformation is based on the assumption that a self-similar solution exists, i.e., every physical parameter preserves its shape during the expansion. Selfsimilar solutions usually describe the asymptotic behavior of an unbounded or a far-field problem; the time $t$ and the space coordinate $x$ appear only in the combination of $f\left(x / t^{\beta}\right)$. It means that the existence of self-similar variables implies the lack of characteristic length and time scales. These solutions are usually not unique and do not take into account the initial stage of the physical expansion process. These kind of solutions describe the intermediate asymptotic of a problem: they hold when the precise initial conditions are no longer important, but before the system has reached its final steady state. They are much simpler than the full solutions and so easier to understand and study in different regions of parameter space. A final reason for studying them is that they are solutions of a system of ODE and hence do not suffer the extra inherent numerical problems of the full partial differential equations. In some cases self-similar solutions help to understand diffusion-like properties or the existence of compact supports of the solution. These analytic solutions can be compared to numerical simulations as well. 
At first we introduce the two dimensional generalization of the self-similar Ansatz, (2.3) which might have the form of

$$
T(x, y, t)=t^{-\alpha} f\left(\frac{F(x, y)}{t^{\beta}}\right),
$$

where $F(x, y)$ could be understood as an implicit parametrization of a onedimensional space curve with continuous first and second derivatives. In our former studies $[5,6]$, we explain that for the non-linearity in the Navier-Stokes equation unfortunately only the $F(x, y)=x+y+c$ function is valid in Cartesian coordinates. It basically comes from the symmetry properties of the system like $u_{x}=u_{y}$. Other locally orthogonal coordinate systems have not been investigated yet.

Every two dimensional flow problem can be reformulated with the help of the stream function $\Psi$ via $u=\Psi_{y}$ and $v=-\Psi_{x}$, which automatically fulfills the continuity equation. The system of (2.2) is now reduced to the following two PDEs

$$
\begin{aligned}
& \Psi_{y t}+\Psi_{y} \Psi_{y x}-\Psi_{x} \Psi_{y y}=-\frac{p_{x}}{\rho}+\left(L \Psi_{y x}\right)_{x}+\left[\frac{L}{2}\left(\Psi_{y y}-\Psi_{x x}\right)\right]_{y} \\
& -\Psi_{x t}-\Psi_{y} \Psi_{x x}+\Psi_{x} \Psi_{x y}=-\frac{p_{y}}{\rho}+\left[\frac{L}{2}\left(\Psi_{y y}-\Psi_{x x}\right)\right]_{x}-\left(L \Psi_{y x}\right)_{y}
\end{aligned}
$$

with $L=\mu_{0}+\mu_{1}\left[2 \Psi_{x y}^{2}+\frac{1}{2}\left(\Psi_{y y}-\Psi_{x x}\right)^{2}\right]^{r / 2}$.

Next, we search the solution of this PDE system such as:

$$
\Psi=t^{-\alpha} f(\eta), \quad p=t^{-\epsilon} h(\eta), \quad \eta=t^{-\beta}(x+y),
$$

where all the exponents $\alpha, \beta, \gamma$ are real numbers. (Solutions with integer exponents are called self-similar solutions of the first kind and they can be obtained from dimensional argumentation as well.)

Unfortunately, the constraints, which should fix the values of the exponents become contradictory, therefore no unambiguous ODE can be formulated. This means that the PDE and the stream function does not have self-similar solutions. In other words the stream function has no diffusive property. This is a very instructive example of the applicability of the trial function of (2.3).

Let's return to the original system of (2.2) and try the ansatz of

$$
u=t^{-\alpha} f(\eta), \quad v=t^{-\delta} g(\eta), \quad p=t^{-\epsilon} h(\eta), \quad \eta=\frac{x+y}{t^{\beta}},
$$

where all the exponents are real number again and $f, g, h$ are called the shape functions. The next step is to determine the exponents. From the continuity equation we simple get arbitrary $\beta$ and $\delta=\alpha$ relations. The two-dimensional NS dictates additional constraints. We skip the trivial case of $\mu_{0} \neq 0, \mu_{1}=0$, which was examined in our former paper as the Newtonian fluid [5]. Finally, we get

$$
\mu_{0}=0, \quad \mu_{1} \neq 0, \quad \alpha=\delta=(1+r) / 2, \quad \beta=(1-r) / 2, \quad \epsilon=r+1 .
$$


Note, that $r$ remains free, which describes various fluids with diverse physical properties, this meets our expectations. For the Newtonian NS equation, there is no such free parameter and in our former investigation we got fixed exponents with a value of $1 / 2$. For a physically relevant solutions, which is spreading and decaying in time all the exponents equations (2.4) have to be positive determining the $-1<r<1$ range. This can be understood as a kind of restricted Ostwald-de Waele-type fluid. After some algebra a second order non-autonomous non-linear ODE remains

$$
\mu_{1}(1+r) f^{\prime \prime}\left[2 f^{\prime}\right]^{r / 2}+\frac{(1-r)}{2} \eta f^{\prime}+\frac{(1+r)}{2} f=0
$$

where prime means derivation with respect to $\eta$. Note that for the numerical value $r=0$ we get back the ODE of the Newtonian NS equation for two dimensions. In three dimensions the ODE reads:

$$
9 \mu_{0} f^{\prime \prime}-3(\eta+c) f^{\prime}+\frac{3}{2} f(\eta)-\frac{c}{2}+a=0 .
$$

Its solutions are the Kummer functions $K U$ and $K M[1]$

$$
f=c_{1} K U\left(-\frac{1}{4}, \frac{1}{2}, \frac{(\eta+c)^{2}}{6 \mu_{0}}\right)+c_{2} K M\left(-\frac{1}{4}, \frac{1}{2}, \frac{(\eta+c)^{2}}{6 \mu_{0}}\right)+\frac{c}{3}-\frac{2 a}{3},
$$

where $c_{1}$ and $c_{2}$ are integration constants, $c$ is the mass flow rate, and $a$ is the external field. These functions have no compact support. The corresponding velocity component however, decays for large time like $v \sim 1 / t$ for $t \rightarrow \infty$, which makes these results physically reasonable. A detailed analysis of (2.6) was presented in [5]. A similar investigation was performed for compressible Newtonian fluids in [6], where the results are given by the Whitakker functions, which have strong connections to the Kummer functions as well.

Unfortunately, we found no analytic solution or integrating factor for (2.5) at any arbitrary values of $r$. For sake of completeness, we mention that with the application of the symmetry properties of the Ansatz $u_{x x}=u_{x y}=u_{y y}=$ $-v_{x x}=-v_{x y}=-v_{y y}$ the following closed form can be derived for the pressure field

$$
h=\rho\left(\mu_{1} 2^{r / 2+1} f^{\prime r+1}+f \eta-\tilde{c}_{1} f\right)+\tilde{c}_{2},
$$

where $\tilde{c}_{1}$ and $\tilde{c}_{2}$ are the usual integration constants.

\section{Phase Plane Analysis}

Applying the transition theorem, a second order ODE is always equivalent to a first order ODE system. Let us substitute $f^{\prime}=l, f^{\prime \prime}=l^{\prime}$, then rewrite (2.5)

$$
\begin{aligned}
f^{\prime} & =l, \\
l^{\prime} & =-\left(\frac{(1-r)}{2} \eta l+\frac{(1+r)}{2} f\right) /\left(\mu_{1}(1+r) 2^{r / 2} l^{r}\right),
\end{aligned}
$$

where prime still means derivation with respect to $\eta$. This ODE system is still non-autonomous and there is no general theory to investigate such phase 
portraits. However, we can divide the second equation of this system by the first one to get a new ODE, where the former independent variable $\eta$ becomes a free real parameter

$$
\frac{d l}{d f}=-\left(\frac{(1-r)}{2} \eta l+\frac{(1+r)}{2} f\right) /\left(\mu_{1}(1+r) 2^{r / 2} l^{r+1}\right) .
$$

In this way we can gain insight into the dynamical properities of the investigated ODE.

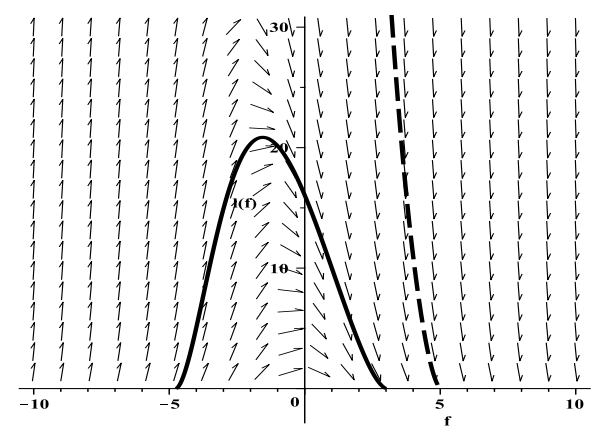

Figure 1. The phase portrait diagram of (3.1) for $\eta=0.03, r=-0.425$ and $\mu_{1}=0.18$. The two different kind of trajectories: one with compact support (solid line), and the other one with compact range (dashed line).

Figure 1 shows the phase portrait diagram of equation (3.1) for water pulp the material parameters are $r=-0.425$ and $\mu_{1}=0.18$. We consider $\eta=0.03$ as the "general time variable" to be positive as well. With the knowledge of the exponent range $-1<r<+1$, two general properties of the phase space can be understood by analyzing equation (3.1).

Firstly, the derivative $d f / d l$ is zero at zero nominator values, which means $l=-(1+r) /(1-r) f / \eta$. This one is a straight line passing through the origin with gradient of $0<(1+r) /(1-r)<\infty$ for $-1<r<+1$. On Figure 1 , the numerical value of the gradient is $-0.403 / \eta=-13.5$.

Secondly, the derivative $d f / d l$ or the direction field is not defined for any negative $l$ values because the power function $l^{-(r+1)}$ in the denominator is not defined for negative $l$ arguments. We can not extract a non-integer root from a negative number. The denominator is always positive.

These properties explain that there are two kinds of possible trajectories or solutions exist in the phase space. One type has a compact support, and the other has a finite range. We may consider the $x$ axis as the velocity $f \sim v(\eta)$ and $y$ axis as the $f^{\prime} \sim a(\eta)$ acceleration for a fixed scaled time $\eta=$ const. It also means that the possible velocity and accelerations for a general time cannot be completely independent from each other. We cannot say that the velocity field or the acccelation field have a compact support but the ratio of them are compact as clearly presented on Figure 1. The factors of the second derivative $f^{\prime \prime}$ show some similarity to the porous media equation, where the 
diffusion coefficient has an exponent also [24]. This is the essential original responsibility for the solution with compact support. This is our main result.

\section{Approximate Solutions}

\subsection{Direct numerical integration}

Using Wolfram Mathematica ${ }^{\circledR} 10$ numerical experiments were made for the Cauchy problem equation (2.5) with $f(0)=0$ and $f^{\prime}(0)=s>0$ and $0<r<1$. It turned out that in all cases there exists an $\eta_{0}>0$ such that $f(\eta)=0$ for $\eta \geq \eta_{0}$. Note, that at the maximum point $\eta_{1}$ of $f(\eta)$ is not differentiable which makes the numerics rather involved. By this reason in the next subsection we approximate using series $f(\eta)$ untill $\eta_{1}$ only.

\subsection{Series expansion}

Our objective is to show the existence of analytic solutions to the differential equation (2.5) and to determine the approximate local solution $f(\eta)$. We use the shooting method and give the conditions at $\eta=0$ with initial conditions

$$
f(0)=A, \quad f^{\prime}(0)=B .
$$

We will consider (2.5) as a system of certain differential equations, namely, the special Briot-Bouquet differential equations. For this type of differential equations we refer to the book by E. Hille [18]. In order to establish the existence of a power series representation of $f(\eta)$ about $\eta=0$ we apply the following theorem.

Briot-Bouquet Theorem [13]: Let us assume that for the system of equations

$$
\left\{\begin{array}{l}
\xi \frac{d z_{1}}{d \xi}=u_{1}\left(\xi, z_{1}(\xi), z_{2}(\xi)\right) \\
\xi \frac{d z_{2}}{d \xi}=u_{2}\left(\xi, z_{1}(\xi), z_{2}(\xi)\right),
\end{array}\right.
$$

where functions $u_{1}$ and $u_{2}$ are holomorphic functions of $\xi, z_{1}(\xi)$, and $z_{2}(\xi)$ near the origin, moreover

$$
u_{1}(0,0,0)=0, \quad u_{2}(0,0,0)=0
$$

then a holomorphic solution of (4.2) satisfying the initial conditions $z_{1}(0)=0$, $z_{2}(0)=0$ exists if none of the eigenvalues of the matrix

$$
\left[\begin{array}{cc}
\left.\frac{\partial u_{1}}{\partial z_{1}}\right|_{(0,0,0)} & \left.\frac{\partial u_{1}}{\partial z_{2}}\right|_{(0,0,0)} \\
\left.\frac{\partial u_{2}}{\partial z_{1}}\right|_{(0,0,0)} & \left.\frac{\partial u_{2}}{\partial z_{2}}\right|_{(0,0,0)}
\end{array}\right]
$$

is a positive integer.

Briot-Bouquet theorem ensures the existence of formal solutions

$$
z_{1}=\sum_{k=0}^{\infty} a_{k} \xi^{k}, \quad z_{2}=\sum_{k=0}^{\infty} b_{k} \xi^{k}
$$


for system (4.2), and also the convergence of formal solutions.

This theorem has been successfully applied to the determination of local analytic solutions of different nonlinear initial value problems [9], [10].

Let us consider the initial value problem (2.5), (4.1) and take its solution in the form

$$
f(\eta)=\eta^{\alpha} Q\left(\eta^{\beta}\right), \eta \in\left(0, \eta_{c}\right),
$$

where function $Q \in C^{2}\left(0, \eta_{c}\right)$ for some positive value $\eta_{c}$. Substituting

$$
f(\eta)=\eta^{\alpha} Q\left(\eta^{\beta}\right)
$$

into (2.5) one can get

$$
\begin{array}{r}
K \frac{1}{\eta}\left\{\beta\left[(\beta+1) \eta^{\beta} Q^{\prime}+\beta \eta^{2 \beta} Q^{\prime \prime}\right]\right\}\left(Q+\beta \eta^{\beta} Q^{\prime}\right)^{\frac{r}{2}} \\
+\frac{1-r}{2} \eta\left(Q+\beta \eta^{\beta} Q^{\prime}\right)+\frac{1+r}{2} \eta Q=0
\end{array}
$$

for $\alpha=1$. Let us introduce the new variable $\xi$ such as $\xi=\eta^{\beta}$ and function $Q$ as follows

$$
Q(\xi)=a_{0}+a_{1} \xi+z(\xi)
$$

where $a_{0}, a_{1}$ are real constants, and $z \in C^{2}\left(0, \eta_{c}^{\beta}\right), z(0)=0, z^{\prime}(0)=0$. We make difference between two cases: $A=0$ and $A \neq 0$.

If $A=0$ then $Q$ fulfills the following properties $Q(0)=a_{0}, Q^{\prime}(0)=a_{1}$, $Q^{\prime \prime}(\xi)=z^{\prime \prime}(\xi)$. From the initial condition $f^{\prime}(0)=Q(0)$ and we have $a_{0}=B$. Applying the Briot-Bouquet theorem, (4.4) yields

$$
\xi z^{\prime \prime}(\xi)=-\frac{1}{\beta}\left[(\beta+1) Q^{\prime}+\xi^{\frac{2}{\beta}-1} \frac{Q+\frac{1-r}{2} \beta \xi Q^{\prime}}{K \beta\left(Q+\beta \xi Q^{\prime}\right)^{\frac{r}{2}}}\right]
$$

with $K=\mu_{1}(1+r) 2^{\frac{r}{2}}$. Therefore, $\beta=2$. We restate the second order differential equation in (4.4) as a system of two equations

$$
\left\{\begin{array}{l}
u_{1}\left(\xi, z_{1}(\xi), z_{2}(\xi)\right)=\xi z_{1}^{\prime}(\xi) \\
u_{2}\left(\xi, z_{1}(\xi), z_{2}(\xi)\right)=\xi z_{2}^{\prime}(\xi)
\end{array}\right.
$$

by choosing

$$
\begin{aligned}
& z_{1}(\xi)=z(\xi), \quad z_{2}(\xi)=z^{\prime}(\xi) \text { with } z_{1}(0)=0, \quad z_{2}(0)=0 \\
& u_{1}\left(\xi, z_{1}(\xi), z_{2}(\xi)\right)=\xi z_{2}, \\
& u_{2}\left(\xi, z_{1}(\xi), z_{2}(\xi)\right)=\xi z_{2}^{\prime}=-\frac{1}{2}\left[3\left(a_{1}+z_{2}\right)+\frac{a_{0}+(2-r) a_{1} \xi+z_{1}+(1-r) \xi z_{2}}{2 K\left(a_{0}+3 a_{1} \xi+z_{1}+2 \xi z_{2}\right)^{\frac{r}{2}}}\right]
\end{aligned}
$$

In order to satisfy the conditions

$$
u_{1}(0,0,0,0)=0, \quad u_{2}(0,0,0,0)=0
$$

in the Briot-Boquet theorem the following connection is obtained

$$
a_{1}=-a_{0}^{1-\frac{r}{2}} /(6 K) .
$$


Therefore, the eigenvalues of matrix $(4.3)$ at $(0,0,0)$ are 0 . Since all eigenvalues are non-positive, referring to the Briot-Bouquet theorem we obtain the existence of unique analytic solutions $z_{1}$ and $z_{2}$ near zero. Thus, there exists a formal solution

$$
f(\eta)=\eta \sum_{k=0}^{\infty} a_{k} \eta^{2 k}
$$

where the first two coefficients are already known. For the determination of coefficients $a_{k}, \quad k>2$, we shall use the J.C.P. Miller formula [19]:

$$
\left[\sum_{k=0}^{L} c_{k} x^{k}\right]^{r / 2}=\sum_{k=0}^{\frac{r}{2} L} d_{k}(r) x^{k}
$$

where $d_{0}(r)=1$ for $c_{0}=1$, and

$$
d_{k}(r)=\frac{1}{k} \sum_{j=0}^{k-1}\left[\frac{r}{2}(k-j)-j\right] d_{j}(r) c_{k-j}, \quad k \geq 1 .
$$

Applying (4.6) we get recursion formula for the determination of $a_{k}$. Comparing the proper coefficients of $\eta$, one can have the values of $a_{k}$ for some $k$; e.g.,

$$
a_{2}=\frac{2-r}{120 K^{2}} a_{0}^{1-r}-\frac{1}{80 K^{2}} a_{0}^{2-r} .
$$

The coefficients are obtained by this method for the power series approximation as

$$
f(\eta)=a_{0} \eta+a_{1} \eta^{3}+a_{2} \eta^{5}+\ldots
$$

Similarly, we can obtain series approximate solution for $A \neq 0$. Here we obtain that $\alpha=0$ and $Q(0)=A=a_{0}, Q^{\prime}(0)=B=a_{1}$ for $f(\eta)=Q\left(\eta^{\beta}\right)$. The differential equation $(2.5)$ yields

$$
K \beta \eta^{\gamma}\left[(\beta-1) Q^{\prime}+\beta \eta^{\beta} Q^{\prime \prime}\right] \beta^{\frac{r}{2}} Q^{\prime \frac{r}{2}}+\frac{1-r}{2} \beta \eta^{\beta} Q^{\prime}+\frac{1+r}{2} Q=0,
$$

where $\gamma=\beta-2+(\beta-1) \frac{r}{2}$. The system of Briot-Bouquet equations is formulated as follows

$$
\left\{\begin{array}{l}
u_{1}\left(\xi, z_{1}(\xi), z_{2}(\xi)\right)=\xi z_{1}^{\prime}(\xi) \\
u_{2}\left(\xi, z_{1}(\xi), z_{2}(\xi)\right)=\xi z_{2}^{\prime}(\xi)
\end{array}\right.
$$

where

$$
\xi z^{\prime \prime}(\xi)=\frac{1-\beta}{\beta} Q^{\prime}-\frac{1-r}{2 K} \xi^{1-\frac{\gamma}{\beta}} \frac{Q^{\prime 1-\frac{r}{2}}}{\beta^{1+\frac{r}{2}}}-\frac{1+r}{2 K} \frac{Q}{\beta^{2+\frac{r}{2}} Q^{\prime \frac{r}{2}}} \xi^{-\frac{\gamma}{\beta}}
$$

The conditions in the Briot-Bouquet theorem can be satisfied for arbitrary $A$ and $B$ if $\gamma=-\beta$, i.e., $\beta=1$. Then we get that

$$
u_{2}\left(\xi, z_{1}(\xi), z_{2}(\xi)\right)=-\frac{1-r}{2 K} \xi^{2}\left(a_{1}+z_{2}\right)^{1-\frac{r}{2}}-\frac{1+r}{2 K} \xi \frac{a_{0}+a_{1} \xi+z_{1}}{\left(a_{1}+z_{2}\right)^{\frac{r}{2}}} .
$$


The method of calculation of the coefficients in the power series is similar to case $A=0$. Equating the like powers one gets the coefficients

$$
a_{2}=-\frac{1+r}{4 K} \frac{a_{0}}{a_{1}^{\frac{r}{2}}}, \quad a_{3}=-\frac{(1+r)^{2} r}{48 K^{2}} \frac{a_{0}^{2}}{a_{1}^{r}}-\frac{a_{1}^{1-\frac{r}{2}}}{6 K}, \ldots
$$

Figure 2 presents the series solution of (2.5) for different values of $r$ when $A=0$ and $B=1$.

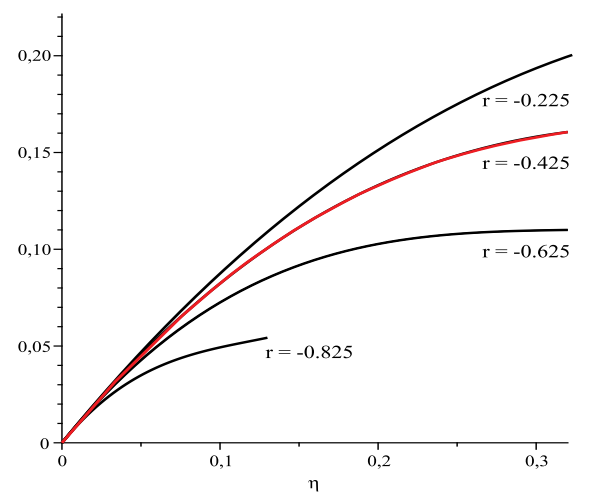

Figure 2. Approximate series solutions of $f(\eta)$ to equation (2.5) and for different values of $r$ when $A=0$ and $B=1$. The red curve is for $r=-0.425$.

Figure 3 presents the series solution of (2.5) for different values of $r$ when $A=1$ and $B=0$. Note, the red curve on both figures presents the solution for $r=-0.425$, which was presented in the phase plane analysis as well. Unfortunatelly, our phase plane and the series expansion results cannot be compared directly.

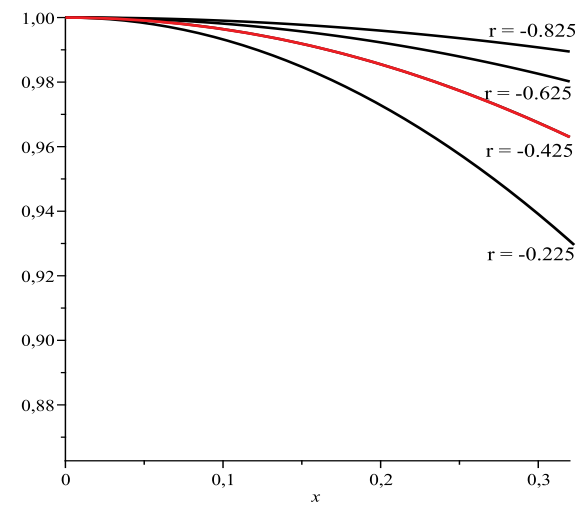

Figure 3. Approximat series solutions of $f(\eta)$ to equation (2.5) and for different values of $r$ when $A=1$ and $B=0$. Note the red curve is for $r=-0.425$. 


\section{Conclusions}

We applied a two-dimensional generalization of the self-similar Ansatz for the Ladyzhenskaya-type non-Newtonian NS equation. We analyzed the final highly non-linear ODE in the phase plane and gave an approximate series expansion solutions as well.

Our main result is that the velocity field of the fluid - in contrast to our former Newtonian result - has a compact support, which is the major difference.

We can explain it with the following everyday example: Let's consider two pots in the kitchen, one is filled with water and the other is filled with chocolate cream. Start to stir both with a wooden spoon in the middle, after a while the whole mass of the water becomes to move due to the Newtonian viscosity, however the chocolate cream far from the spoon remains stopped even after a long time.

\section{Acknowledgement}

This work was supported by the Hungarian OTKA NK 101438 Grant. We thank for Prof. Robert Kersner for useful discussions and comments. We dedicate this paper to our spouses.

The research work presented in this paper is based on the results achieved within the TÁMOP-4.2.1.B- 10/2/KONV-2010-0001 project and carried out as part of the TÁMOP-4.1.1.C-12/1/KONV-2012-0002 "Cooperation between higher education, research institutes and automotive industry" project in the framework of the New Széchenyi Plan. The realization of this project is supported by the Hungarian Government, by the European Union, and co-financed by the European Social Fund.

\section{References}

[1] M. Abramowitz and I.A. Stegun. Handbook of Mathematical Functions. Dover Publications, Inc., New York, 1968.

[2] G. Astarita and G. Marucci. Principles of non-Newtonian fluid mechanics. McGraw-Hill, London, New York, 1974.

[3] H-O. Bae and H.J. Choe. Theory of non-Newtonian flow. Trends in Mathematics Inf. Cent. for Math. Sci., 1:201-208, 1998.

[4] G.I. Barenblatt. Similarity, self-similarity, and intermediate asymptotics. Consultants Bureau, New York, London, 1979.

[5] I.F. Barna. Self-similar solutions of three-dimensional Navier-Stokes equation. Communications in Theoretical Physics, 56(4):745-750, 2011. http://dx.doi.org/10.1088/0253-6102/56/4/25.

[6] I.F. Barna and L. Mátyás. Analytic solutions for the three-dimensional compressible Navier-Stokes equation. Fluid Dynamics Research, 46(5), 2014. http://dx.doi.org/10.1088/0169-5983/46/5/055508.

[7] N. Bedjaoui, M. Guedda and Z. Hammouch. Similarity solutions of the Rayleigh problem for Ostwald-de Wael electrically conducting fluids. Analysis and Applications, 9(02):135-159, 2011. http://dx.doi.org/10.1142/S0219530511001789. 
[8] M. Benlahsen, M. Guedda and R. Kersner. The generalized Blasius equation revisited. Mathematical and Computer Modelling, 47(9-10):1063-1076, 2008. http://dx.doi.org/10.1016/j.mcm.2007.06.019.

[9] G. Bognár. Local analytic solutions to some nonhomogeneous problems with p-Laplacian. Electronic Journal of Qualitative Theory of Differential Equations, 4:1-8, 2008.

[10] G. Bognár. Similarity solution of boundary layer flow for non-Newtonian fluids. International Journal of Nonlinear Sciences and Numerical Simulation, 10:15551566, 2009. http://dx.doi.org/10.1515/IJNSNS.2009.10.11-12.1555.

[11] G. Bognár. Analytic solutions to the boundary layer problem over a stretching wall. Computers $\mathscr{E}$ Mathematics with Applications, 61(8):2256-2261, 2011. http://dx.doi.org/10.1016/j.camwa.2010.09.039.

[12] G. Böhme. Non-Newtonian Fluid Mechanics. North-Holland Series in Applied Mathematics and Mechanics. Amsterdam: North-Holland, 1987.

[13] Ch. Briot and J.K. Bouquet. Étude des fonctions d'une variable imaginaire. Journal de l'École Polytechnique, Cashier, 36:85-131, 1856.

[14] D.X. Du, Y.G. Li and S.J. Sun. Pseudo-plastic behavior of a film foam flow with carbon dioxide as the internal gas phase. Advanced Materials Research, 221:15-20, 2011. http://dx.doi.org/10.4028/www.scientific.net/AMR.221.15.

[15] M. Guedda. Similarity and pseudosimilarity solutions of degenerate boundary layer equations. Handbook of Differential Equations: Stationary Partial Differential Equations, 4:117-202, 2007. http://dx.doi.org/10.1016/S18745733(07)80006-4.

[16] M. Guedda and Z. Hammouch. Similarity flow solutions of a non-Newtonian power-law fluid. International Journal of Nonlinear Science, 6(3):255-264, 2008.

[17] M. Guedda and R. Kersner. Non-Newtonian pseudoplastic fluids: Analytical results and exact solutions. International Journal of Non-Linear Mechanics, 46(7):949-957, 2011. http://dx.doi.org/10.1016/j.ijnonlinmec.2011.04.009.

[18] E. Hille. Ordinary Differential Equations in the complex domain. John Wiley \& Sons, New York-London-Sydney, 1976.

[19] L. Howarth. On the solution of the laminar boundary layer equations. Proceedings of the Royal Society of London A: Mathematical, Physical and Engineering Sciences, 164(919):547-579, 1938. http://dx.doi.org/10.1098/rspa.1938.0037.

[20] A.M. Ishak, J.H. Merkin, R. Nazar and I. Pop. Mixed convection boundary layer flow over a permeable vertical surface with prescribed wall heat flux. Zeitschrift für angewandte Mathematik und Physik, 59(1):100-123, 2008. http://dx.doi.org/10.1007/s00033-006-6082-7.

[21] O.A. Ladyzhenskaya. Boundary Value Problems of Mathematical Physics. Amer. Math. Soc. Providence, RI, 1970.

[22] L. Sedov. Similarity and Dimensional Methods in Mechanics. CRC Press, Boca Raton, Florida, 1993.

[23] D.H. Wei and S. Al-Ashhab. Similarity solutions for non-newtonian power-law fluid flow. Applied Mathematics and Mechanics, 35(9):1155-1166, 2014.

[24] Ya. B. Zel'dovich and A.S. Kompaneets. Collection dedicated to the 70th birthday of A.F. Joffe. Izdat. Akad. Nauk. SSSR, 1950.

[25] Ya.B. Zel'dovich and Yu.P. Raizer. Physics of Shock Waves and High Temperature Hydrodynamic Phenomena. Academic Press, New York, 1966. 